\begin{tabular}{|c|c|c|c|c|}
\hline \multicolumn{5}{|c|}{ НАУЧНО-ТЕХНИЧЕСКИЙ ВЕСТНИК ИНФОРМАЦИОННЫХ ТЕХНОЛОГИЙ, МЕХАНИКИ И ОПТИКИ } \\
\hline 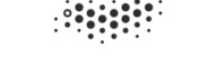 & $\begin{array}{ll}\text { июль-август } 2018 & \text { Том } 18 \text { № } 4 \\
\text { SCIENTIFIC AND TECHNICAL JOURNAL } 0\end{array}$ & $\begin{array}{l}\text { ISSN 2226-1494 } \\
\text { NFORMATION TEC }\end{array}$ & $\begin{array}{l}\text { http://ntv.ifmo.rul } \\
\text { IECHANICS AND OPTICS }\end{array}$ & 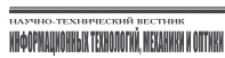 \\
\hline УНИВЕРСИТЕТ ИТМО & July-August 2018 & ISSN 2226-1494 & http://ntv.ifmo.ru/en & \\
\hline
\end{tabular}

УДК 004.89; 004.942

МОДЕЛИРОВАНИЕ ПРОЦЕССОВ ПОДБОРА ПАРАМЕТРОВ ОБЛАЧНЫХ СИСТЕМ ДЛЯ ОБЕСПЕЧЕНИЯ ИХ УСТОЙЧИВОСТИ С УЧЕТОМ НАДЕЖНОСТИ И БЕЗОПАСНОСТИ

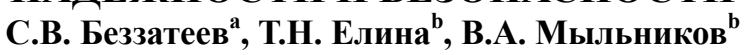

${ }^{\text {a }}$ Университет ИТМО, Санкт-Петербург, 197101, Российская Федерация

b Санкт-Петербургский государственный университет аэрокосмического приборостроения, Санкт-Петербург, 190000 , Российская Федерация

Адрес для переписки: bsv@aanet.ru

Информация о статье

Поступила в редакцию 21.05 .18 , принята к печати 25.06 .18

doi: 10.17586/2226-1494-2018-18-4-654-662

Язык статьи - русский

Ссылка для цитирования: Беззатеев С.В., Елина Т.Н., Мыльников В.А. Моделирование процессов подбора параметров облачных систем для обеспечения их устойчивости с учетом надежности и безопасности // Научно-технический вестник информационных технологий, механики и оптики. 2018. Т. 18. № 4. С. 654-662. doi: 10.17586/2226-1494-2018-18-4-654-662

\title{
Аннотация
}

Проведен анализ коммерческого и свободного программного обеспечения для организации облачных вычислений, определены достоинства и недостатки существующих методов повышения надежности и безопасности вычислительных систем. Большинство существующих систем не учитывает ряд факторов, влияющих на безопасность, надежность и производительность вычислений, сложность адаптации к изменяющимся требованиям и условиям внешней среды. Сформулирована цель работы, заключающаяся в подборе архитектуры системы облачных вычислений, обеспечивающей максимальное удовлетворение заявок с различным уровнем приоритета, поступающих как от пользователей, так и от сервисов самой системы. Для решения данной задачи предложена методика конфигурирования системы облачных сервисов, основанная на модели нейро-нечеткой системы, позволяющая повысить производительность выполнения запросов пользователей при обеспечении надежности и безопасности обрабатываемой информации в системах специального и двойного назначения. Разработана архитектура нейронечеткой сети, определены ее входные и выходные параметры. В качестве примера с помощью предложенных моделей проведено конфигурирование облачной информационной системы, предназначенной для решения определенных групп задач. Результатом решения явилась матрица распределения ресурсов системы для обслуживания различных групп задач.

Ключевые слова

облачные вычисления, виртуализация, критерии безопасности, нейро-нечеткие сети, генетический алгоритм

\section{MODELING OF SELECTION PROCESSES OF CLOUD SYSTEMS PARAMETERS PROVIDING THEIR STABILITY IN ACCORDANCE WITH RELIABILITY AND SAFETY}

\section{S.V. Bezzateev ${ }^{a}$, T.N. Elina ${ }^{b}$, V.A. Myl'nikov ${ }^{b}$}

a ITMO University, Saint Petersburg, 197101, Russian Federation

${ }^{\mathbf{b}}$ Saint Petersburg State University of Aerospace Instrumentation, Saint Petersburg, 190000, Russia Federation

Corresponding author: bsv@aanet.ru

Article info

Received 21.05.18, accepted 25.06.18

doi: 10.17586/2226-1494-2018-18-4-654-662

Article in Russian

For citation: Bezzateev S.V., Elina T.N., Myl'nikov V.A. Modeling of selection processes of cloud systems parameters providing their stability in accordance with reliability and safety. Scientific and Technical Journal of Information Technologies, Mechanics and Optics, 2018, vol. 18, no. 4, pp. 654-662 (in Russian). doi: 10.17586/2226-1494-2018-18-4-654-662

\section{Abstract}

We have carried out the analysis of commercial and free software for support and organization of cloud computing, outlined the advantages and disadvantages of existing methods for reliability and security improvement of computing systems. Most 
of the existing systems do not take into account a number of factors that affect the safety, reliability and performance of calculations, the complexity of adaptation to changing requirements and environmental conditions. The work objective is formulated consisting in selection of cloud computing system architecture that provides maximum satisfaction of requests with different priority level, coming both from users and from services of the system itself. To solve this problem we propose a method of the system configuring for cloud services based on the model of a neuro-fuzzy system. The method gives the possibility to increase the productivity of users' requests providing the reliability and security of the processed information in special-purpose and dual-use systems. The architecture of the neuro-fuzzy network is developed, its input and output parameters are determined. Applying the proposed models, the configuration of a cloud information system designed to solve certain groups of tasks is carried out as an example. The decision result was a distribution matrix of system resources for serving of different task groups.

Keywords

cloud computing, virtualization, security criteria, neural-fuzzy networks, genetic algorithm

\section{Введение}

В настоящее время имеется большой выбор коммерческого и свободного программного обеспечения для организации облачных вычислений, позволяющего обеспечить масштабируемость, удобство сопровождения, высокую безопасность, надежность и производительность. Это дает возможность создавать пользовательскую информационную инфраструктуру для обработки данных с учетом требуемых характеристик надежности, безопасности и специфики деятельности организации [1]. К недостаткам существующих методов конфигурирования облачных систем относится игнорирование многих факторов, влияющих на безопасность, надежность и производительность вычислений, сложность адаптации к изменяющимся требованиям и условиям внешней среды, контроля и распределения ресурсов для задач с различными требованиями надежности, безопасности и оперативности на одном вычислительном узле [2]. Примером могут служить изолированные друг от друга сервисы различных клиентов, где каждый имеет индивидуальные графики используемых информационных ресурсов с явно выраженным равномерным и пиковым поведением нагрузки.

Обеспечение надежности и безопасности, имеющее наивысший приоритет, требует дополнительных расходов на общесистемном уровне, а это может привести к снижению оперативности и функциональности клиентских задач [3]. Критической ситуацией является возникновение отказа и восстановление работоспособности инфраструктуры после его устранения.

Сервисы клиентов существенно отличаются по типу используемых ресурсов. Не существует универсального вычислительного узла, обладающего требуемыми характеристиками безопасности и надежности для всех задач, у каждого узла есть сильные и слабые звенья [4]. Часто решения по перераспределению сервисов принимаются с учетом опыта персонала по прогнозированию нештатных ситуаций.

Существующие методы повышения надежности и безопасности вычислительных систем основаны на резервировании элементов, таких как жесткий диск, канал связи, сервер, однако при этом не рассматривается проблема существования критически важных факторов, которые приводят к дестабилизации и отказу работы всей системы [5-9]. В работе [10] предложен метод повышения надежности облачных систем путем внедрения гомогенности, позволяющий рассчитать минимальное число узлов с учетом показателей надежности. Однако в упомянутых работах отсутствует анализ влияния различных предшествующих активных и пассивных состояний серверов в момент возникновения отказа на момент восстановления. Например, отказ элемента на одном сервере в различной степени влияет на активное состояние элементов других взаимодействующих с ним серверов кластера.

\section{Постановка задачи исследования}

Для учета большого числа как количественных, так и качественных характеристик, при отсутствии явной статистической корреляции между исходными факторами и параметрами надежности, безопасности и производительности системы, а также для обеспечения возможности гибкой адаптации архитектуры системы с элементами диагностики и прогнозирования, авторами предлагается использование аппарата нейро-нечетких систем [11] для моделирования процессов эксплуатации облачных сервисов.

Предлагается следующий состав ключевых параметров для формирования архитектуры облачного сервиса.

1. Модель развертывания облака: частная/гибридная.

2. Набор функциональных задач.

3. Качественные показатели облака: уровень безопасности данных; уровень масштабируемости системы; степень автоматизированного самообслуживания системы со стороны провайдера; наличие системы широкого сетевого доступа через стандартные механизмы для различных платформ, тонких и толстых клиентов; сосредоточенность ресурсов на отдельных площадках для их эффективного распределения; уровень эластичности, который позволяет почти мгновенно предоставлять в любом размере требуемые вычислительные ресурсы под конкретные условия задачи; наличие системы управления облаком с автоматическим контролем и оптимизацией выделяемых ресурсов. 
4. Время отклика системы на запрос пользователя.

5. Интенсивность поступающих запросов.

6. Степень загрузки вычислительных узлов.

7. Интенсивность межмашинного взаимодействия.

Предлагается следующий набор выходных данных, задающих архитектуру и характеристики системы.

1. Характеристики организационной модели облачной инфраструктуры: с централизованным хранилищем и (или) распределенной файловой системой.

2. Характеристики программно-аппаратного обеспечения: характеристики вычислительных узлов; допустимые параметры виртуальных машин по используемым ресурсам; характеристики устройств внешней памяти; характеристики внешнего и внутреннего сетевого взаимодействия; типы сервисов; предлагаемое программное обеспечение.

Постановка задачи заключается в подборе архитектуры системы облачных вычислений [12], обеспечивающей максимальное удовлетворение заявок с различным уровнем приоритета, поступающих как от пользователей, так и от сервисов самой системы.

Важной дополнительной характеристикой для классификации клиентских сервисов является поддержка мультизадачности. В рамках настоящей работы данный термин определяет влияние увеличения числа процессоров на рост производительности сервиса. При слабой поддержке мультизадачности или ее отсутствии производительность сервиса больше зависит от тактовой частоты процессора вычислительного узла.

Предлагается разделить все заявки на группы в зависимости от их приоритета и требуемого типа ресурсов, а в качестве критерия оптимальности использовать коэффициент загрузки системы $\left(K_{3}\right)$, определяемый как доля ресурсов, задействованная в обслуживании заданий:

$$
K_{3}=\frac{1}{I J} \frac{\sum_{i=1}^{I} \sum_{j=1}^{J} \mathbf{M}_{i j}^{\phi} \mathbf{V} \mathbf{I}_{i} \mathbf{V I} \mathbf{I}_{j}}{\sum_{i=1}^{I} \sum_{j=1}^{J} \mathbf{M}_{i j}^{p}} \rightarrow 1,
$$

где $i=1, \ldots, I-$ номер группы задач; $j=1, \ldots, J$ - номер вида ресурса; $\mathbf{M}_{i j}^{\phi}$ и $\mathbf{M}_{i j}^{p}$ - матрицы фактического и расчетного распределения ресурсов системы; VI $\mathbf{I}_{i} \in(0 ; 1)$ - вес (важность) группы заявок $i$, оцениваемый экспертным путем; $\mathbf{V I}_{j} \in(0 ; 1)$ - вес (важность) группы ресурсов $j$, определяемый отношением стоимости ресурса к стоимости владения системой.

Для целевой функции (1) определены ограничения (2)-(8), касающиеся используемых системой ресурсов: стоимость владения системой (Cost), ресурсы процессора по группам задач $\left(\right.$ Proc $\left._{i}\right)$ представленные в виде списка характеристик логических ядер для поддержки мультизадачности, ресурсы оперативной и постоянной памяти $\left(R A M_{i}, H D D_{i}\right)$, сетевые ресурсы $\left(N e t_{i}\right)$, ограничения по обеспечению требуемой степени надежности системы $\left(\operatorname{Rel}_{i}\right)$ и безопасности обрабатываемых данных $(\mathrm{Saf} i)$.

$$
\begin{aligned}
& \operatorname{Cost}^{\text {расч }} \leq \operatorname{Cost}^{\text {треб }}, \\
& \operatorname{Proc}_{i}^{\text {расч }} \in\left(\operatorname{Proc}_{i}^{\min } \ldots \operatorname{Proc}_{i}^{\max }\right) \cdot k_{\mathrm{pe} 3}^{\text {Proc }}, \\
& R A M_{i}^{\text {pacч }} \in\left(R A M_{i}^{\min } \ldots R A M_{i}^{\max }\right) \cdot k_{\mathrm{pe} 3}^{R A M}, \\
& H D D_{i}^{\text {pacч }} \in\left(H D D_{i}^{\min } \ldots H D D_{i}^{\max }\right) \cdot k_{\mathrm{pe} 3}^{H D D}, \\
& \operatorname{Net}_{i}^{\text {pacч }} \in\left(N e t_{i}^{\min } \ldots N e t_{i}^{\max }\right) \cdot k_{\mathrm{pe} 3}^{N e t}, \\
& \operatorname{Re}_{i}^{\text {pacч }} \geq \operatorname{Rel}_{i}^{\text {треб }}, \\
& \operatorname{Saf}_{i}^{\text {pacч }} \geq \operatorname{Saf}_{i}^{\text {треб }},
\end{aligned}
$$

где индексы «расч», «треб» - указатель на расчетное и требуемое значение критерия; max и min - указатели на максимальное и минимальное требуемое значение критерия соответственно; $k_{\text {рез }}-$ коэффициенты резерва для обеспечения бесперебойной работы системы по видам ресурсов с учетом требований надежности и безопасности.

Требуемые критерии безопасности обрабатываемых данных $\left(\mathrm{Saf}_{i}\right)$ определяются в соответствии с руководящим документом ${ }^{1}$ и применяются к гостевым виртуальным средам в индивидуальном порядке. Каждый из критериев соответствует выполнению группы требований и активирует требования предыдущего уровня:

\footnotetext{
1 Руководящий документ №187 от 19.06 .2002 г. Безопасность информационных технологий. Критерии оценки безопасности информационных технологий.
} 
- низкий - выполнение базовых требований безопасности;

- средний - обеспечение контроля доступа, антивирусная проверка файловых ресурсов;

- высокий - сканирование сетевых протоколов, организация локальных или внешних межсетевых экранов;

- очень высокий - проактивная защита, изолированная среда выполнения приложений, контроль устройств, регистрация событий в сеансе пользователя.

Требуемая степень надежности $\left(R e l_{i}\right)$ представляется как интегральный показатель готовности информационной системы и рассчитывается по методике [13]. Каждый из критериев соответствует выполнению группы требований:

- низкий - время простоя определяется длительностью восстановления виртуальной среды или обрабатываемых данных из резервной копии, используется локальная файловая система вычислительного узла;

- средний - время простоя определяется длительностью перезагрузки виртуальной среды на исправном вычислительном узле, требуется распределенная файловая система;

- высокий - время простоя определяется длительностью онлайн-миграции виртуальной среды на исправный вычислительный узел, требуется распределенная файловая система, требуется репликация образа файловой системы и оперативной памяти виртуальной среды, требуется поддержка функционала отказоустойчивого кластера.

Выполнение требований показателей качества тесно связано с организационной моделью облачной инфраструктуры и характеристиками аппаратного обеспечения. На скорость технологических операций оказывает существенное влияние способ виртуализации (полная виртуализация, паравиртуализация, виртуализация уровня операционной системы), организация распределенной файловой системы между узлами вычислительного кластера и т.д.

Для выполнения требований безопасности и надежности могут потребоваться дополнительные ресурсы. При ограниченных ресурсах возрастает риск потери устойчивости. Ситуация с отключением и восстановлением одного из видов оборудования может привести к различным последствиям, поэтому необходимо заранее планировать регламентные работы по обслуживанию инфраструктуры [14]. Это заставляет вносить дополнительные корректировки в виде коэффициента резерва $\left(k_{\text {рез }}\right)$ по каждому из ресурсов. Значение коэффициента показывает увеличение ресурса из-за технологических операций при организации облачной инфраструктуры и рассчитывается по следующему выражению:

$$
\begin{aligned}
& k_{\mathrm{pe} 3}^{P r o c}=\frac{\operatorname{Proc}_{\text {вирт }}+P r o c_{\text {техн }}}{P r o c_{\text {вирт }}}, k_{\text {ре3 }}^{R A M}=\frac{R A M_{\text {вирт }}+R A M_{\text {техн }}}{R A M_{\text {вирт }}}, \\
& k_{\text {ре3 }}^{H D D}=\frac{H D D_{\text {вирт }}+H D D_{\text {техн }}}{H D D_{\text {вирт }}}, k_{\text {ре3 }}^{\text {Net }}=\frac{N e t_{\text {вирт }}+N e t_{\text {техн }}}{N e t_{\text {вирт }}},
\end{aligned}
$$

где $k_{\text {рез }}$ - коэффициенты резерва по видам ресурсов; индекс «вирт» - указатель на используемые ресурсы всех виртуальных сред; индекс «техн» - указатель на используемые ресурсы для организации функционирования виртуальных сред.

Результатом решения данной оптимизационной задачи является матрица распределения ресурсов системы (М) для обслуживания различных групп заявок:

$$
\mathbf{M}=\left(\begin{array}{cccc}
\operatorname{Proc}_{11} & \operatorname{Proc}_{12} & \cdots & \operatorname{Proc}_{1 I} \\
R A M_{21} & R A M_{22} & \cdots & R_{2 I} \\
H D D_{31} & H D D_{32} & \cdots & H D D_{3 I} \\
\text { Net }_{j 1} & \text { Net }_{j 2} & \cdots & \text { Net }_{J I}
\end{array}\right) .
$$

\section{Нейро-нечеткая модель распределения ресурсов облачного сервиса}

Для построения нейро-нечеткой сети на основе выделенных ранее количественных и качественных критериев были построены соответствующие лингвистические переменные, определены их термы и функции принадлежности. На предварительном этапе в качестве функций принадлежности использованы типовые $L-R$-функции: треугольные, трапецеидальные и сигмоидного типа.

В качестве алгоритма нечеткого вывода для сети использован адаптированный алгоритм Цукамото. На вход сети подается матрица $\mathbf{x} \in\left\{x_{i j}\right\}$, где $x_{i j}$ - значение показателя $j$ по группе заявок $i$.

Шаг 1. Введение нечеткости (этап фаззификации): определение степени срабатывания (истинности) каждой предпосылки каждого правила $k$ для заданных значений входных переменных

$$
f_{k}(x), k=1, \ldots, K .
$$

Шаг 2. Агрегирование степеней истинности предпосылок по каждому из правил $\alpha_{k}$ :

$$
\alpha_{k}=\min \left\{\mu_{A_{i j}}(x)\right\} \text {. }
$$


Шаг 3. Активизация заключений по каждому из правил:

$\beta_{k}=f_{k}^{-1}\left(\alpha_{k}\right)$.

В результате находятся четкие значения выходных переменных в каждом из заключений правил.

Шаг 4. Этап аккумулирования активизированных заключений правил в данном алгоритме отсутствует вследствие четких значений выходных переменных.

Шаг 5. Дефаззификация методом центра тяжести для одноточечных множеств:

$$
x=\frac{\sum_{k=1}^{K} \alpha_{k} \beta_{k}}{\sum_{k=1}^{K} \alpha_{k}} .
$$

В нечетких продукционных моделях наблюдается экспоненциальное возрастание числа нечетких правил при стремлении к нулю ошибки аппроксимации. Задача заключается в поиске компромисса между требуемой точностью и числом правил модели, которые позволяют ее достичь за приемлемое время.

Число нечетких правил с конъюнктивной формой предпосылки, необходимых для покрытия пространства входных переменных, определяется как декартово произведение лингвистических термов входных переменных. С целью минимизации этой величины выполнена декомпозиция входных переменных на модели с меньшим числом входных переменных в базе правил с последующим их объединением в многослойную структуру.

Структура пятислойной нечеткой продукционной сети, реализующей алгоритм нечеткого вывода Цукамото, представлена на рис. 1. Входами данной модели являются исходные данные для проектирования архитектуры системы облачных вычислений, а выходами - значения элементов матрицы М.

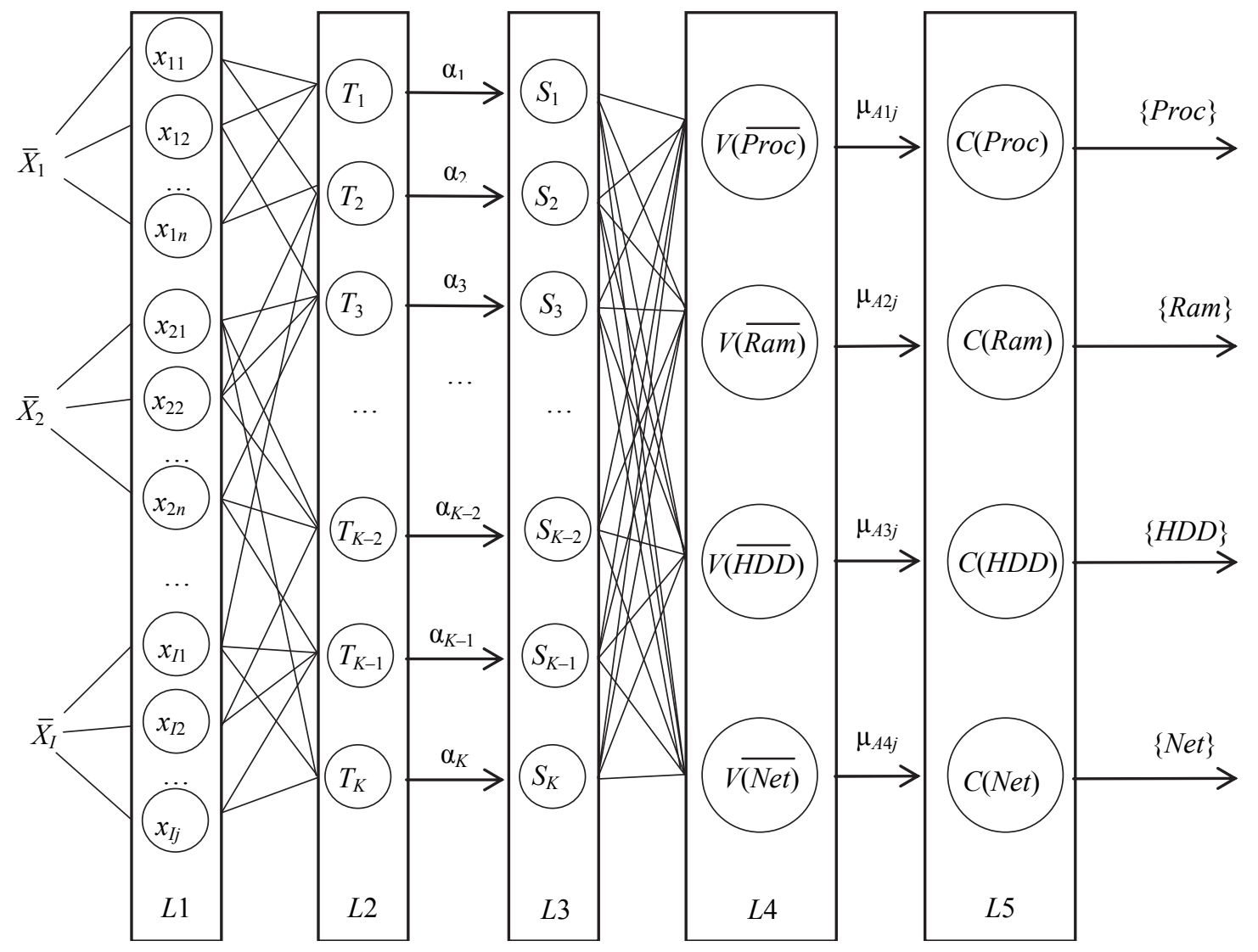

Рис. 1. Структура нейро-нечеткой сети

Слой $L 1$. Включает элементы, реализующие функцию принадлежности нечеткого множества $A_{i j}$. Их выходы - значения функций принадлежности $\mu_{A_{j i}}(x)$ при конкретных (заданных) значениях входных переменных $x$. Цель слоя - оценить степень принадлежности входных данных $x$ к соответствующим нечетким множествам $A_{j i}$.

Слой $L 2$. Конфигурация связей этого слоя соответствует базе правил, а мультипликаторы - блоку вывода. Элементы второго слоя выполняют агрегирование степеней истинности предпосылок каждого 
правила базы в соответствии с операцией $T$-нормы, в качестве которой здесь используется операция minконъюнкции [15]. Каждый узел слоя $L 1$ соединен со всеми узлами слоя $L 2$ и представляет $k$-ое правило с соответствующими нечеткими множествами суждений этого правила.

Слой L3. Элементы данного слоя выполняют активизацию (определение степеней истинности) заключений по каждому из правил на основе операции тіn-активизации [8]. Количество элементов этого слоя равно количеству элементов слоя $L 2$. Каждый узел слоя $L 3$ связан с узлом слоя $L 2$ соответствующего $k$-го правила. На выходе слоя формируется результат вывода в виде значения функции принадлежности $\alpha_{k}$-уровня $-\mu_{A_{i j}} k$-го правила.

Слой $L 4$. Содержит четыре узла, которые связаны со всеми узлами слоя $L 3$. Элементы данного слоя аккумулируют полученные на предыдущем этапе заключения по всем правилам. Объединение найденных усеченных нечетких множеств проводится с использованием операции максимум (maxдизъюнкции).

Слой $L 5$. Элементы этого слоя вычисляют выход сети, который определяется как «центр тяжести» для фигуры $\mu_{A_{i j}}(x)$ - этап приведения к четкости (defuzzification) выходной переменной $y$.

Процесс обучения нейро-нечеткой сети состоял в уточнении форм функций принадлежности, их центров и параметров, а также в коррекции нечетких правил в процессе наблюдения за объектом и уменьшения их количества путем объединения.

Для корректной настройки параметров функций принадлежности входов и выходов, проведено обучение нейро-нечеткой сети с помощью эволюционного алгоритма, состоящего из двух этапов. На первом этапе выбрана схема представления весов связей в виде двоичных последовательностей с шагом $r$ $=10^{-6}$ на интервале $[0,1]$.

На втором этапе на популяции особей (множестве весов нейронной сети) реализован генетический алгоритм, упрощенная схема которого представлена на рис. 2.

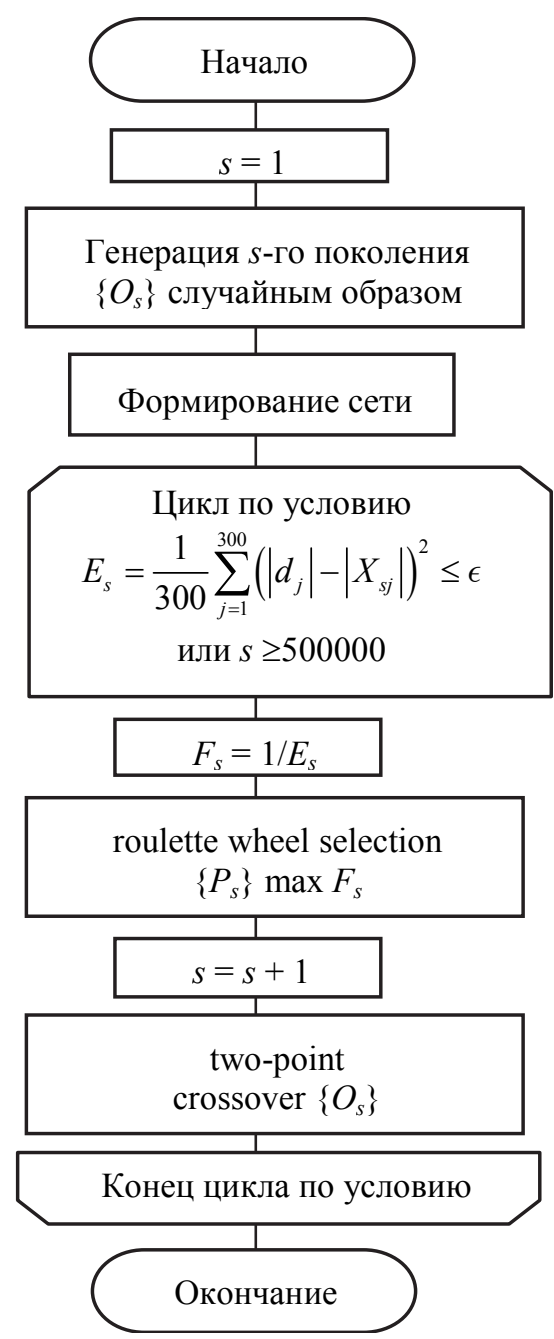

Рис. 2. Укрупненный алгоритм обучения нейро-нечеткой сети 
На рис. 2: $\left\{O_{s}\right\}-s$-ое поколение особей численностью 180 , каждая из которых состоит из 30 хромосом, что соответствует количеству настраиваемых параметров сети; $E_{s}-$ общая среднеквадратическая погрешность между фактическими $d_{j}$ и заданными $x_{s j}$ значениями на выходах сети при подаче на ее входы обучающих образов; $F_{s}$ - функция приспособленности $s$-ой особи; $\left\{P_{s}\right\}-s$-ое поколение родительской популяции численностью 180; roulette wheel selection и two-point crossover - применяемые методы селекции и скрещивания соответственно.

В результате обучения сети скорректированы параметры функций принадлежности входных и выходных переменных, что позволило достичь результатов расчетов нейро-нечеткой сети, близких к экспертным оценкам по сравнению с необученной сетью.

\section{Результаты конфигурирования облачной системы}

В качестве примера проведено конфигурирование облачной информационной системы, предназначенной для решения следующих групп задач.

Задача 1. Проведение бухгалтерских расчетов в среде конфигураций «1С:Предприятие» с использованием терминального сервера доступа.

Задача 2. Ведение корпоративного файлового хранилища.

Задача 3. Веб-сервис обслуживания клиентов электронного магазина.

Расчеты проводились в трех вариантах: оценки экспертов (I), необученная сеть (I), обученная сеть (III). Результаты сравнительных расчетов приведены в таблице. Для оценки процессоров использовалась оценка производительности в синтетических тестах. Стоимость системы определяется архитектурой и типом серверной платформы и может существенно различаться, несмотря на схожие характеристики синтетических тестов. Расхождения в стоимости зависят от характеристик надежности разработчика серверной платформы и устойчивости работы системы при различных пиковых нагрузках, поэтому мнение эксперта может идти в сторону увеличения владения системой.

\begin{tabular}{|c|c|c|c|c|c|}
\hline \multirow{2}{*}{\multicolumn{2}{|c|}{$\begin{array}{l}\text { Оценки } \\
\text { показателей }\end{array}$}} & \multicolumn{4}{|c|}{ Группы задач } \\
\hline & & Задача 1 & Задача 2 & Задача 3 & Вычислительный узел \\
\hline \multirow{3}{*}{$K_{3}$, o.e. } & $\mathrm{I}$ & 0,82 & 0,73 & 0,87 & 0,88 \\
\hline & II & 0,74 & 0,66 & 0,78 & 0,69 \\
\hline & III & 0,84 & 0,71 & 0,86 & 0,89 \\
\hline \multirow{3}{*}{$\begin{array}{l}\text { Cost, } \\
\text { y.e. }\end{array}$} & $\mathrm{I}$ & 84626 & 44080 & 90960 & 163800 \\
\hline & II & 88220 & 50380 & 80160 & 184522 \\
\hline & III & 82220 & 45380 & 82100 & 155440 \\
\hline \multirow{3}{*}{$\begin{array}{l}\text { Proc, } \\
\text { синт. } \\
\text { тест }\end{array}$} & $\mathrm{I}$ & $\begin{array}{c}2 \times 16302 \\
(\text { Intel Xeon E5-2623 v3) }\end{array}$ & $\begin{array}{c}2 \times 4114 \\
\text { (Intel Xeon } \\
\text { L3110) } \\
\end{array}$ & $\begin{array}{c}4 \times 6268 \\
(\text { Intel Xeon X3230) }\end{array}$ & $\begin{array}{c}2 \times 32190 \\
\text { (Intel Xeon E7-4870 } \\
\text { v2) } \\
\end{array}$ \\
\hline & II & $\begin{array}{c}2 \times 16140 \\
(\text { Intel Xeon E5-2643) }\end{array}$ & $\begin{array}{c}2 \times 4057 \\
(\text { Intel Xeon } \\
\text { E3110) } \\
\end{array}$ & $\begin{array}{c}4 \times 6096 \\
(\text { Intel Xeon X3430) }\end{array}$ & $\begin{array}{c}2 \times 31748 \\
\text { (Intel Xeon E5-2687W } \\
\text { v3) }\end{array}$ \\
\hline & III & $\begin{array}{c}2 \times 16140 \\
(\text { Intel Xeon E5-2643) }\end{array}$ & $\begin{array}{c}2 \times 4035 \\
\text { (Intel Xeon } \\
\text { E3110) } \\
\end{array}$ & $\begin{array}{c}4 \times 6221 \\
(\text { Intel Xeon X3320) }\end{array}$ & $\begin{array}{c}4 \times 16140 \\
(\text { Intel Xeon E5-2643) }\end{array}$ \\
\hline \multirow{3}{*}{$\begin{array}{l}\text { RAM, } \\
\text { ГБ }\end{array}$} & $\mathrm{I}$ & 10 & 4 & 12 & 24 \\
\hline & II & 12 & 4 & 14 & 28 \\
\hline & III & 9 & 4 & 12 & 25 \\
\hline \multirow{3}{*}{$\begin{array}{l}H D D, \\
\text { ТБ }\end{array}$} & $\mathrm{I}$ & 0,5 & 10 & 0,5 & 12 \\
\hline & II & 0,8 & 8 & 0,6 & 10 \\
\hline & III & 0,4 & 10,5 & 0,6 & 12,5 \\
\hline \multirow{3}{*}{$\begin{array}{l}\text { Net, } \\
\text { Кбит/с }\end{array}$} & $\mathrm{I}$ & 100 & 1000 & 1000 & 1000 \\
\hline & II & 150 & 850 & 850 & 1200 \\
\hline & III & 120 & 950 & 1100 & 1050 \\
\hline \multirow{3}{*}{ Rel } & $\mathrm{I}$ & Высокая & Высокая & Очень высокая & Очень высокая \\
\hline & II & Очень высокая & Средняя & Высокая & Очень высокая \\
\hline & III & Очень высокая & Высокая & Очень высокая & Очень высокая \\
\hline \multirow{3}{*}{ Saf } & $\mathrm{I}$ & Высокая & Средняя & Высокая & Высокая \\
\hline & II & Средняя & Высокая & Высокая & Высокая \\
\hline & III & Высокая & Средняя & Высокая & Высокая \\
\hline
\end{tabular}

Таблица. Результаты конфигурирования облачной информационной системы 
Результаты проведенных расчетов (таблица) позволяют сделать вывод о небольшом расхождении показателей, сформированных экспертным путем и нейро-нечеткой системой. Расхождение данных первого и третьего варианта не превышает $20 \%$, а в среднем составляет $7 \%$. Для правильного обучения сети требуется достаточный объем статистических данных о производительности системы, что позволит уменьшить данное расхождение.

\section{Заключение}

Использование нейро-нечеткой экспертной системы для конфигурирования архитектуры облачных сервисов позволит предприятию оптимально распределять вычислительные ресурсы по различным группам решаемых задач и экономить денежные средства на их приобретение без привлечения профессионалов экспертного класса, а также обеспечить высокий уровень надежности, безопасности и производительности обработки данных. Дальнейшее развитие системы направлено на решение задач оперативного перераспределения ресурсов в рамках одного предприятия в зависимости от изменяющейся структуры поступающих в систему задач.

\section{Литература}

1. Зацаринный А.А., Сучков А.П. Система ситуационного управления как мультисервисная технология в облачной среде // Информатика и ее применения. 2018. № 1. С. 78-88. doi: $10.14357 / 19922264180110$

2. Tsai J.M., Hung S.W. A novel model of technology diffusion: system dynamics perspective for cloud computing // Journal of Engineering and Technology Management. 2014. V. 33. P. 47 62. doi: 10.1016/j.jengtecman.2014.02.003

3. Li J., Naughton J.F., Nehme R.V. Resource bricolage and resource selection for parallel database systems // VLDB Journal. 2017. V. 26. N 1. P. 31-54. doi: 10.1007/s00778-0160435-4

4. Грушо А.А., Забежайло М.И., Зацаринный А.А. Контроль и управление информационными потоками в облачной среде // Информатика и ее применения. 2015. Т. 9. № 4. С. 91-97. doi: $10.14357 / 1992264150410$

5. Singh P., Dutta M., Aggarwal N. A review of task scheduling based on meta-heuristics approach in cloud computing / Knowledge and Information Systems. 2017. V. 52. N 1. doi: 10.1007/s10115-017-1044-2

6. Гудкова И.А., Масловская Н.Д. Вероятностная модель для анализа задержки доступа к инфраструктуре облачных вычислений с системой мониторинга // Т-Comm: Телекоммуникации и транспорт. 2014. № 6. С. 13-15.

7. Мокров Е.В., Чукарин А.В. Анализ показателей эффективности системы облачных вычислений с миграцией серверов // Т-Сomm: Телекоммуникации и транспорт. 2014 № 8. С. 64-67.

8. Горбунова А.В., Зарядов И.С., Матюшенко С.И., Самуйлов К.Е., Шоргин С.Я. Аппроксимация времени отклика системы облачных вычислений // Информатика и ее применения. 2015. Т. 9. № 3. С. 32-38. doi: $10.14357 / 19922264150304$

9. Stukalova A.A., Guskov A.E. Publications on the use of cloud technologies at libraries // Scientific and Technical Information Processing. 2016. V. 43. N 1. P. 47-57. doi: $10.3103 / \mathrm{S} 0147688216010093$

10. Полторак В.П., Троцкий С.А. Метод повышения надежности информационных телекоммуникационных облаков путем внедрения гомогенности // Вісник НТУУ КПІ. Сер. Радіотехніка. Радіоапаратобудування. 2012. № 51 C. $97-105$.

11. Leroux S., Bohez S., De Coninck E., Verbelen T., Vankeirsbilck B., Simoens P., Dhoedt B. The cascading neural network: building the internet of smart things // Knowledge and Information Systems. 2017. V. 52. N 3. P. 791-814. doi 10.1007/s10115-017-1029-1

12. Елин Н.Н., Фомичева С.Г., Елина Т.Н., Мыльников В.А Моделирование процессов управления проектами на основе мультиагентных информационных технологий // Известия вузов. Технология текстильной промышленности. 2016. № 5(365). С. 220-224.

13. Газуль С.М., Бабаев Э.О., Горнов П.А. Интегральный показатель готовности информационной системы к работе в облаке // Международный научно-исследовательский

\section{References}

1. Zatsarinny A.A., Suchkov A.P. The situational management system as a multiservice technology in the cloud. Informatics and Applications, 2018, no. 1, pp. 78-88. (in Russian) doi: $10.14357 / 19922264180110$

2. Tsai J.M., Hung S.W. A novel model of technology diffusion: system dynamics perspective for cloud computing. Journal of Engineering and Technology Management, 2014, vol. 33, pp. 47-62. doi: 10.1016/j.jengtecman.2014.02.003

3. Li J., Naughton J.F., Nehme R.V. Resource bricolage and resource selection for parallel database systems. VLDB Journal, 2017, vol. 26, no. 1, pp. 31-54. doi: 10.1007/s00778-016-0435-

4. Grusho A.A., Zabezhailo M.I., Zatsarinny A.A. Information flow monitoring and control in the cloud computing environment. Informatics and Applications, 2015, vol. 9, no. 4 pp. 91-97. (in Russian) doi: 10.14357/1992264150410

5. Singh P., Dutta M., Aggarwal N. A review of task scheduling based on meta-heuristics approach in cloud computing. Knowledge and Information Systems, 2017, vol. 52, no. 1. doi: 10.1007/s10115-017-1044-2

6. Gudkova I.A., Maslovskaya N.D. Probability model for analysing impact of delays due to monitoring on mean service time in cloud computing. T-Comm: Telecommunications and Transport, 2014, no. 6, pp. 13-15. (in Russian)

7. Mokrov E.V., Chukarin A.V. Performance analysis of cloud computing system with live migration. T-Comm: Telecommunications and Transport, 2014, no. 8, pp. 64-67. (in Russian)

8. Gorbunova A.V., Zaryadov I.S., Matyushenko S.I., Samujlov K.E., Shorgin S.Ya. The approximation of responsw time of a cloud computing system. Informatics and Applications, 2015, vol. 9, no. 3, pp. 32-38. (in Russian) doi: $10.14357 / 19922264150304$

9. Stukalova A.A., Guskov A.E. Publications on the use of cloud technologies at libraries. Scientific and Technical Information Processing, 2016, vol. 43, no. 1, pp.47-57. doi: 10.3103/S0147688216010093

10. Poltorak V.P., Trotskij S.A. Method for enhancing the reliability of information telecommunication clouds through the introduction of homogeneity. Visnyk NTUU KPI Seriia Radiotekhnika Radioaparatobuduvannia, 2012, no. 51, pp. 97 105. (in Russian)

11. Leroux S., Bohez S., De Coninck E., Verbelen T. Vankeirsbilck B., Simoens P., Dhoedt B. The cascading neural network: building the internet of smart things. Knowledge and Information Systems, 2017, vol. 52, no. 3, pp. 791-814. doi: 10.1007/s10115-017-1029-1

12. Elin N.N., Fomicheva S.G., Elina T.N., Myl'nikov V.A Modeling of project management processes based on multiagent information technology. Izvestiya vuzov. Tekhnologiya Tekstil'noi Promyshlennosti, 2016, no. 5, pp. 220-224. (in Russian)

13. Gazul S.M., Babaev E.O., Gornov P.A. The integral index of the readiness of the information system to work in a calculating 
журнал. 2014. № 4-2. С. 14-16.

14. Мыльников В.А., Елина Т.Н. Повышение оперативности и надежности облачной инфраструктуры на базе распределенной файловой системы // Актуальные вопросы естествознания: материалы III Всероссийской научнопрактической конференции с международным участием. Иваново, 2018. С. 266-268.

15. Елина Т.Н., Абалдова С.Ю. Нейро-нечеткое моделирование процессов оценки результативности системы менеджмента качества // Известия вузов. ЭФиУП. 2014. № 2(20). C. $111-118$.

\section{Авторы}

Беззатеев Сергей Валентинович - доктор технических наук, доцент, заведующий кафедрой, Университет ИТМО, СанктПетербург, 197101, Российская Федерация, Scopus ID: 6602425996, ORCID ID: 0000-0002-0924-6221, bsv@aanet.ru

Елина Татьяна Николаевна - кандидат экономических наук, доцент, доцент, Санкт-Петербургский государственный университет аэрокосмического приборостроения, СанктПетербург, 190000, Российская Федерация, Scopus ID: 7801649822, ORCID ID: 0000-0001-5221-7621, elinatn@yandex.ru Мыльников Владимир Аркадьевич - кандидат технических наук, доцент, доцент, Санкт-Петербургский государственный университет аэрокосмического приборостроения, СанктПетербург, 190000, Российская Федерация, Scopus ID: 57194518638, ORCID ID: 0000-0002-7532-9607, va.myinikov@yandex.ru cloud. International Research Journal, 2014, no. 4-2, pp. 14-16. (in Russian)

14. Myl'nikov V.A., Elina T.N. Increase the efficiency and reliability of the cloud infrastructure based on a distributed file system. Actual Problems of Natural Science: Proc. $3^{\text {rd }}$ AllRussian Conf. Ivanovo, Russia, 2018, pp. 266-268.

15. Elina T.N., Abaldova S.Yu. Neuro-fuzzy modeling of assessing the effectiveness of quality management system. News of Higher Educational Institutions. Series Economy, Finance and Production Management, 2014, no. 2, pp.111-118. (in Russian)

\section{Authors}

Sergey V. Bezzateev - D.Sc., Associate Professor, Head of Chair, ITMO University, Saint Petersburg, 197101, Russian Federation, Scopus ID: 6602425996, ORCID ID: 0000-0002-0924-6221, bsv@aanet.ru

Tatyana N. Elina - PhD, Associate Professor, Associate Professor, Saint Petersburg State University of Aerospace Instrumentation, Saint Petersburg, 190000, Russia Federation, Scopus ID: 7801649822, ORCID: 0000-0001-5221-7621, elinatn@yandex.ru

Vladimir A. Myl'nikov - PhD, Associate Professor, Associate Professor, Saint Petersburg State University of Aerospace Instrumentation, Saint Petersburg, 190000, Russia Federation, Scopus ID: 57194518638, ORCID: 0000-0002-7532-9607, va.myinikov@yandex.ru 